\title{
Das vergessene Geschlecht
} The Forgotten Gender

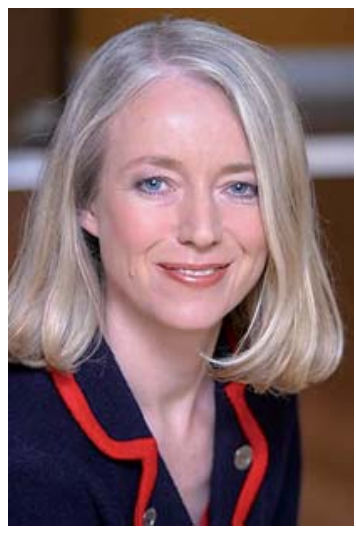

Prof. Dr. Christiane Bayerl

\section{Bibliografie}

Dol http://dx.doi.org/ 10.1055/s-0029-1244175

Akt Dermatol 2010; 36; 201

(c) Georg Thieme Verlag KG . Stuttgart · New York

ISSN 0340-2541

\section{Korrespondenzadresse}

Prof. Dr. med. Christiane BayerI

Klinik für Dermatologie und

Allergologie, HSK,

Wilhelm-Fresenius-Klinik GmbH

Städtisches Klinikum Wiesbaden

Lehrkrankenhaus der

Universität Mainz

Aukammallee 39

65191 Wiesbaden

christiane.bayerl@

hsk-wiesbaden.de
Das starke Geschlecht stecke in der Sinnkrise - so ein Spiegelartikel. Es beginne schon früh. Jungs seien zumeist den Mädchen in der Schule unterlegen. Sie seien weniger flexibel, häufiger krank und antworteten auf Irritationen mit Gewalt. Wie schaut es aus mit der Haut des Mannes?

Männerhaut enthält nicht mehr Talgdrüsen, aber größere. Daher kommt es zu einer stärkeren Talgproduktion. Androgene erhöhen die Drüsenfunktion. Der Hydrolipidmantel ist üblicherweise stabil. Die Verhornung ist bei Männerhaut stärker. Die Epidermis ist um 15-24\% dicker als bei Frauen. Die Faltenbildung setzt später ein, dafür sind die Falten tiefer. Die Männerhaut ist intensiv durchblutet, was jedoch mit zunehmendem Alter deutlicher zurückgeht als bei Frauen. Der pH-Wert liegt üblicherweise im sauren Bereich bei 5,4. Männerhaut ist dunkler pigmentiert als Frauenhaut.

Während das Geschlecht eines Kindes bei Geburt üblicherweise determiniert ist, ist es der Hauttyp nicht. Die Einteilung der Hauttypen ist umstritten. Dennoch hat sich eine Klassifikation in normale Haut, empfindliche/hypersensitive Haut, trockene Haut und seborrhoische, zu Akne neigende Haut eingebürgert. Etwa $80 \%$ der pubertierenden jungen Männer entwickeln zumindest vorübergehend eine seborrhoische Haut bis hin zu einer manifesten Akne. Der Akne-Behandlungsalgorithmus ist hinlänglich publiziert und die neu überarbeiteten Leitlinien liegen vor. Aus kosmetologischer Sicht kann begleitend behandelt werden, z.B. mit Chemical Peeling oder bestimmten topischen Zubereitungen, die die Haut beruhigen. Für irritierte Männerhaut werden adstringierend wirkende Extrakte, z. B. aus Baumrinden oder Gerbsäure-haltige Inhaltsstoffe, eingesetzt. Sinnvoll sind auch mild schälende Inhaltsstoffe, keratolytische Substanzen wie Salizylsäurederivate, Fruchtsäure, Glykolsäure oder Milchsäure. Die In-vitro-Studien bei der Suche nach Externa für die Männerhaut überprüfen üblicherweise die Hemmung der Lipoxygenase, die Inhibition von Entzündungsmediatoren und die Reduktion von Ödem und Vasodilatation.

Trockene Haut wird von Männern beklagt nach Hitze, Wind oder Kälteexposition oder bei trockener Raumluft. Klimaanlagen und Flugreisen verschlechtern die Sympotmatik. Dazu kommt die mechanische Irritation am Hemdkragen und durch Rasur. Mangelnde Rückfettung ist durchaus typisch, denn „Mann“ cremt nicht gerne.
Die empfindliche, hypersensitive Haut ist ein Symptom mit endogenen und exogenen Ursachen. In Asien werden als Ursache scharfe Gewürze und Temperaturschwankungen angegeben, in Amerika der Wind und in Europa die Kosmetika. 50\% der Frauen geben an eine empfindliche, hypersensitive Haut zu haben; bei den Männern sind es immerhin $40 \%$. Beklagt werden Juckreiz, Brennen, Stechen und ein Spannungsgefühl an der Haut mit oder ohne objektivierbaren Befund. Messmethoden sind der Stinging-Test nach Kligman mit 5-10\%-iger Milchsäure nasolabial, die pH-Messung und die Bestimmung des transepidermalen Wasserverlustes. Aber es existiert keine exakte Definition über Messparameter. Die beliebteste „Nachweismethode“ dürfte wohl der Rotweinprovokationstest sein, der sich in der Tat in der Fachliteratur findet. Bei empfindlicher, hypersenisitiver Haut ist Vorsicht geboten beim topischen Einsatz von Fruchtsäuren, Salizylsäuren, Retinoiden, Urea, Vitamin C und Propylenglykol u.ä. Aktive Substanzen bei trockener und empfindlicher Haut sollten schützen und den Wiederaufbau der Hautbarriere fördern. In vitro werden Marker der epidermalen Differenzierung untersucht wie Filaggrin etc. Natural Moisturizing Factors sind sinnvoll; ebenso Inhaltsstoffe oder Partikel, die mechanische Reibung reduzieren. Dabei darf die Zubereitung nicht fetten, nicht den Hemdkragen verfärben und muss schnell einziehen. $\mathrm{Zu}$ beachten ist, dass Männer bei den Duftstoffallergien aufgeholt haben und fast so häufig sensibilisiert sind wie Frauen.

Männerhaut ist zwar etwas „dicker“ als Frauenhaut, fettiger aufgrund der größeren Talgdrüsen und dennoch gereizter durch Krägen und Rasur. Insofern sind komedogene Inhaltsstoffe zu meiden. Unter beruhigenden Inhaltsstoffen wird oft ein kühlender Effekt auf die Haut verstanden. Die Tools das zu messen haben wir, z.B. Thermografieteste mit der Infrarotkamera.

Das Anti-Aging-Präparat für den Mann unterscheidet sich nicht von den Empfehlungen für die Damenwelt. Nummer eins ist nach wie vor der UVAund UVB-Schutz. Topische Vitamin-A-Säurederivate wie Retinol und Retinaldehyde sind in der Kosmetologie die Inhaltsstoffe mit der größten Evidenz. Männer fragen vermehrt beim Dermatologen wegen kosmetologischer Empfehlungen nach. Ein Training in der richtigen Hautpflege für den Mann ist eine Aufgabe für uns Dermatologen. Produkte mit guter Verträglichkeit und Wirkung sind vonnöten. Sie müssen für den Mann passen. 